\title{
Role of signal transducer and activator of transcription 3 in liver fibrosis progression in chronic hepatitis C-infected patients
}

\author{
Peter Stärkel, Christine De Saeger, Isabelle Leclercq and Yves Horsmans
}

In vitro and animal data suggest that hepatitis $\mathrm{C}$ virus $(\mathrm{HCV})$ proteins might interfere with signal transducer and activator of transcription 3 (Stat3) signaling. It remains unknown whether Stat3 influences the apoptotic-proliferation balance and how this may relate to liver fibrosis progression in HCV-infected patients. We assessed Stat3 expression and DNA-binding as well as expression of its regulators protein inhibitor of activated Stat 3 (Pias3) and suppressor of cytokine signaling 3 (Socs3) in $65 \mathrm{HCV}$-infected livers at various stages of fibrosis progression. We then determined the level of expression of the proliferation markers cyclin D1 and proliferating cell nuclear antigen (PCNA) in conjunction with pro- and antiapoptotic markers Bax and Bcl-2 in the same liver samples. With the onset of fibrosis, Stat3 DNA-binding decreased and became almost undetectable in livers with bridging fibrosis or cirrhosis. Stat3 DNA-binding inversely correlated with Pias3 expression and Stat3-Pias3 interaction increased with the progression of fibrosis. Cyclin D1 and PCNA in hepatocytes decreased dramatically during fibrosis progression and levels highly correlated with Stat3 expression. In addition, an antiapoptotic profile due to upregulation of $\mathrm{Bcl}-2$ principally in infiltrating inflammatory cells was observed with progressing fibrosis. In conclusion, fibrosis progression is characterized by a continuous decline in Stat3 DNA-binding activity related to overexpression and progressive interaction of Pias3-Stat3. The decrease in Stat3 activity correlated with reduced hepatocytes proliferation and a positive antiapoptotic balance in infiltrating inflammatory cells that are known mediators of cell damage in HCV.

Laboratory Investigation (2007) 87, 173-181. doi:10.1038/labinvest.3700496; published online 27 November 2006

KEYWORDS: HCV; Stat3; fibrosis; proliferation; apoptosis

Over the last years, evidence is accumulating in favor of a direct relationship between the hepatitis $\mathrm{C}$ virus (HCV) and intracellular mechanisms under the control of signal transducer and activator of transcription 3 (Stat3) molecules. In vitro data suggest that proteins of the $\mathrm{HCV}$ interfere with Stat3 signaling. ${ }^{1-4}$ Recent publications also underscore the increasing interest in Stat3 concerning the pathogenesis of HCV-related disease and antiviral response mechanisms. ${ }^{5,6}$ However, most of these studies were performed in cell culture or replicon systems that cannot easily be extrapolated to human disease. To date, clear evidence that Stat3 is implicated in the pathogenesis and progression of HCV-related liver disease in vivo in humans is still lacking. In addition, there is no clear consensus to whether the evolution of chronic liver disease towards fibrosis and ultimately to cirrhosis results from decreased hepatocytes proliferation, increased apoptosis or a combination of both phenomenon. $^{7-11}$ Stat3 may play a pivotal role in the progression of HCV-related liver disease as it controls intracellular signal transduction pathways of several proinflammatory cytokines and growth factors implicated in liver damage and repair mechanisms. ${ }^{12-15}$ In particular, disruption of Stat3-mediated intracellular signal transduction pathways leads to disturbed liver regeneration and repair ${ }^{16,17}$ and may therefore constitute a bridge between HCV infection, deficient cellular proliferation and the occurrence and progression of fibrosis. We previously reported that Stat 3 activity is disturbed in human end-stage alcoholic and HCV-related cirrhosis. ${ }^{18,19}$ It remains, however, to be investigated whether alterations in Stat3 activity occur gradually during disease progression and are implicated in the pathogenesis of HCV-related liver disease or whether they should only be considered as a by-

Department and Laboratory of Gastroenterology, St Luc University Hospital, Université Catholique de Louvain, Brussels, Belgium Correspondence: Dr P Stärkel, MD, PhD, Department of Gastroenterology, St Luc University Hospital, Av. Hippocrate 10, 1200 Brussels, Belgium. E-mail: Peter.Starkel@gaen.ucl.ac.be 
stander of end-stage liver disease. In addition, the potential relationship between Stat3 alterations and cell proliferation or apoptosis has not been clarified in human liver. Hepatitis $\mathrm{C}$ infection represents a suitable model to assess many of these open questions since intermediate stages of disease progression have been defined and liver tissue may be obtained from liver biopsies that are routinely performed during the clinical work-up of the disease. We hypothesize that fibrosis progression in $\mathrm{HCV}$-induced liver disease may be related to a progressive alteration in Stat 3 function that might ultimately alter the capacity of liver to regenerate and repair itself. To test this hypothesis, we assessed Stat3 expression and function in liver samples from HCV-infected patients at various stages of fibrosis progression. Changes in Stat 3 were then correlated with changes in the expression of proliferation and apoptosis markers in the same liver samples. We show that a progressive protein inhibitor of activated Stat 3 (Pias3) induced reduction in Stat3 activity is likely implicated in the pathogenesis of the disease from viral infection to occurrence of fibrosis and progression towards cirrhosis in vivo in human liver samples. In addition, we present evidence that the progressive decrease of Stat3 activity is associated with a gradual reduction of hepatocytes proliferation and an increased antiapoptotic response in infiltrating lymphocytes. These observations suggest not only a mechanistic and pathogenic link between Stat 3 and both phenomenons but also add new data to the hypothesis of cirrhosis development being a consequence of deficient cell proliferation.

\section{MATERIALS AND METHODS Patients and Liver Samples}

Liver tissue was obtained from patients undergoing liver biopsy for standard work-up of HCV infection. In addition, small cubes of liver tissue were removed from explanted livers of HCV patients at transplantation. Surplus tissue from size-reduced liver grafts was used as histologically normal HCV-negative liver specimens $(n=6)$. Tissue samples were immediately frozen in liquid nitrogen and stored at $-80^{\circ} \mathrm{C}$ until use. Only surplus tissue that was not required for pathological analysis anymore was used for the study. Consequently, due to the very limited amount of tissue available from liver biopsies, the specimens were divided into two series of samples: one series was used for mRNA studies and Stat3 DNA-binding experiments and the second one was designated to immunoprecipitation and protein expression studies. As a consequence, correlations can only be established for the paired analysis of various factors in each series. Overall HCV samples from 65 patients have been used for this study. The number of HCV-positive specimens $(n)$ used to perform the various analyses is indicated in the respective graphs and figures. All tissue samples were examined by an experienced liver pathologist and scored according to the Ludwig's scoring system. ${ }^{20}$ Samples with high grade of portal and or lobular inflammation (P2L2, P3L1-3, P1-3L3) were discarded to minimize the impact of tissue inflammation on the expression and activity of the various factors examined in the study. Samples were matched for low portal (P) and lobular (L) inflammation scores (P1L2 or P2L1) in order to assure homogeneity and to focus on the variation of fibrosis as the principal pathological factor. According to the fibrosis score, the specimens were subdivided into three groups: no fibrosis (S1), fibrotic septas extending from the portal tracts (S2), bridging fibrosis and/or established cirrhosis $(\mathrm{S} 3 / 4)$.

\section{Preparation of Cellular Extracts}

Liver homogenates were prepared as previously described. ${ }^{18}$ Whole liver cell extracts were obtained from frozen tissues using a commercially available extract kit as indicated by the manufacturer (Active Motif, Rixensart, Belgium). Protein concentrations were determined using a BCA Protein Assay (Pierce Chemical, Rockford, IL, USA).

\section{RNA Isolation}

Total RNA was prepared from frozen liver tissue using TriPure Isolation Reagent (Roche Diagnostics, Mannheim, Germany).

\section{Immunoblotting}

Immunoblotting was performed according to standard techniques. The membranes were revealed with primary and secondary antibodies as summarized in Table 1.

Protein expression was quantified by densitometry using the Gel Doc 2000 system and soft ware (Bio-Rad Laboratories, Nazareth, Belgium) and normalized for their respective $\beta$-actin levels (loading controls). Three identical samples were systematically loaded on each membrane in order ensure homogeneity and allow comparisons between the membranes. Membranes were stripped $\left(\right.$ Restore $^{\mathrm{TM}}$ Western Blot Stripping Buffer, Pierce Chemical) and reprobed whenever possible.

\section{Immunoprecipitation}

The Seize ${ }^{\circledR}$ X Protein A immunoprecipitation Kit (Pierce Chemical) was used to for immunoprecipitation studies according to the instructions of the manufacturer.

\section{Immunohistochemistry}

Immunohistochemical staining of proliferating cell nuclear antigen (PCNA) on liver slides was performed as previously described. ${ }^{21}$ A similar procedure was used for Bcl-2 and Bax staining, except that slides were heated in a $10 \mathrm{mM}$ citrate buffer $\mathrm{pH} 5.7$ for $75 \mathrm{~min}$ for optimal antigen retrieval. Antibodies and working conditions are depicted in Table 1.

\section{Stat3 DNA-Binding Activity Assay}

The TransAM ${ }^{\mathrm{TM}}$ Stat3 transcription factor assay kit (Active Motif) was used to determine Stat3 DNA-binding activity following the instructions of the manufacturer. 
Table 1 Antibodies and working conditions used for WB and IH

\begin{tabular}{lll}
\hline Antibody & Working conditions & Source \\
\hline Stat3 rabbit polyclonal & $1: 2500,1 \mathrm{~h}$ & Santa Cruz Biotechnology, Santa Cruz, CA, USA \\
Phospho-Stat3 Y705 rabbit polyclonal & $1: 1500,2 \mathrm{~h}$ & Upstate Biotechnology, Lake Placid, NY, USA \\
BCl-2 mouse monoclonal & $1: 500$, overnight (WB) & Santa Cruz \\
& $1: 200$, overnight (IH) & Dako, Glostrup, Denmark \\
BCl-xl mouse monoclonal & $1: 500$, overnight & Santa Cruz \\
Bax mouse monoclonal & $1: 500$, overnight (WB) & Santa Cruz \\
& $1: 500$, overnight (IH) & Dako \\
Cyclin D1 mouse monoclonal & $1: 2000,2 \mathrm{~h}$ & Santa Cruz \\
PCNA mouse monoclonal & $1: 750$, overnight (WB) & Dako \\
Peroxidase-conjugated goat anti-rabbit and goat anti-mouse lgG & $1: 20000$ to 1:50 000; $1 \mathrm{~h}$ & Jackson ImmunoResearch, West Grove, PA, USA \\
\hline
\end{tabular}

WB, Western blotting; $I \mathrm{H}$, immunohistochemistry.

\section{Reverse Transcription and Real-Time Polymerase Chain Reaction}

As previously reported in detail, ${ }^{18}$ total liver RNA was reverse-transcribed and analyzed by quantitative polymerase chain reaction (PCR) (GeneAmp ${ }^{\mathbb{R}} 5700$ Sequence Detection System and software, Applied Biosystems, Den Ijssel, Netherlands) according to standard methods and the standardized thermal profile of the system set by the manufacturer. Primers were designed using the Primer Express ${ }^{\mathrm{TM}}$ design software (Applied Biosystems) and are summarized in Table 2.

Quantifications were obtained based on the $\Delta C_{\mathrm{T}}$ values as indicated by the manufacturer.

\section{Statistical Analysis}

Results are expressed as means \pm standard error of the mean (s.e.m.). Statistical differences between the groups were tested using the Student's $t$-test. The Spearman nonparametric test was used to examine correlations. Statistical significance was assumed for a $P$-value $<0.05$.

\section{RESULTS}

The Impact of Viral Infection on the Expression of Stat3 and Stat3 Regulators Compared to Normal HCV-Negative Livers

As a first step, we examined whether the presence of the virus does modify the expression of Stat 3 and its regulators. Therefore, liver samples from HCV-infected patients without evidence of fibrosis on histology and minimal inflammation scores were compared to histological normal HCV-negative liver samples.

Viral infection elicited a fivefold decrease in Stat3 mRNA expression compared to noninfected normal livers $(0.21 \pm 0.03$ vs $1.03 \pm 0.14, P<0.001)$. However, total Stat3 and phospho-Stat3 protein expression were only slightly reduced in HCV-infected livers compared to normal
Table 2 Primers used for quantitative PCR

\begin{tabular}{lll}
\hline Primer & Sense & Antisense \\
\hline Stat3 & ggaggaggcattcggaaag & atctgtgtgacaccaacga \\
Pias3 & tgtccagtctccactacatgagta & ttcagacagagagtcagcactatgg \\
SOCS-3 & ttcagcatctctgtcggaagac & cggcagctgggtgacttt \\
RPL19 & caagcggattctcatggaaca & tggtcagccaggagcttctt \\
\hline
\end{tabular}

ones (Figure 1). Pias3, an inhibitor of Stat3 DNA-binding in the nucleus, ${ }^{22}$ was weakly expressed in normal livers. Viral infection did not affect Pias3 mRNA expression compared to normal liver samples $(1.23 \pm 0.14$ vs $1.55 \pm 0.25, P=\mathrm{NS})$ but slightly increased Pias3 protein levels (Figure 1). Stat3 DNAbinding decreased by $50 \%$ in $\mathrm{HCV}$-infected livers compared to normal livers $(0.117 \pm 0.037 v s 0.297 \pm 0.014, P<0.001)$ in association with reduced Stat 3 and increased Pias 3 protein expression. Suppressor of cytokine signaling 3 (Socs3) mRNA expression, a Stat3 regulator interfering with Stat3 phosphorylation via Jaks, ${ }^{23,24}$ dropped significantly in HCV-infected livers in comparison to normal liver samples $(1.35 \pm 0.35$ vs $18,94 \pm 2.49, P<0.001)$ making it unlikely that Socs3 accounts for the reduction in Stat3 DNA-binding observed in HCV-infected livers.

\section{Fibrosis Progression in HCV-Infected Livers is Associated with an Additional Highly Significant Decline in Stat3 \\ Function}

Viral infection seems to interfere with Stat3 function in HCV-infected nonfibrotic livers as indicated by our observations above. Given the potential role of Stat3 in liver repair mechanisms, we were prompted to investigate whether the occurrence and progression of fibrosis is associated with a 


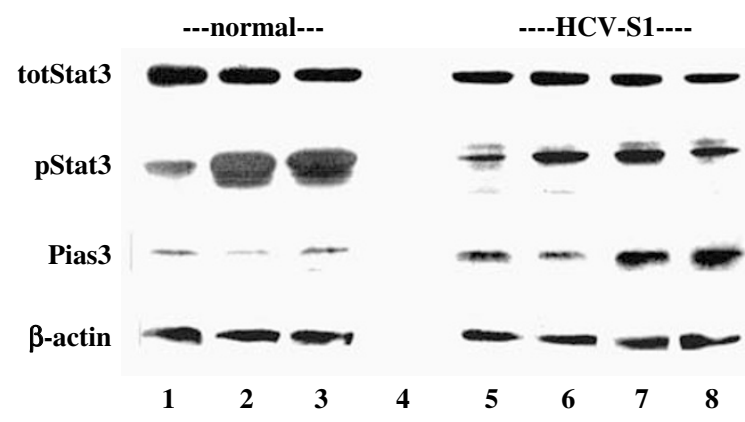

Figure 1 Representative Western blots of total (totStat3) and phosphorylated (pStat3) Stat3, and Pias3 expression in histologically normal $\mathrm{HCV}$-negative livers compared to HCV-infected livers without fibrosis (S1). $\beta$-Actin represents the respective loading controls.

further decline in Stat3 function in carefully matched HCV-infected liver samples with various degrees of fibrosis.

\section{Fibrosis progression reduces Stat 3 expression and DNA-binding activity}

Compared to its expression in nonfibrotic HCV-infected livers, Stat3 mRNA expression increased again with the onset of fibrosis (S2) and remained high in established cirrhosis (S3/4) (Figure 2a). In opposite to the mRNA, total Stat3 protein expression decreased further by $25 \%$ with the onset of fibrosis. This reduction became highly significant in S3/4 HCV-infected livers (50\% decrease) suggesting that Stat3 is mainly regulated at the protein level (Figure $2 \mathrm{~b}$ ). By contrast, phospho-Stat3 expression remained unaffected by the occurrence of liver fibrosis (Figure 2b). Interestingly, an additional decrease in Stat3 DNA-binding activity by $40 \%$ was found in HCV-infected livers with the onset of fibrosis compared to HCV livers without fibrosis. Stat3 DNA-binding activity further declined ( $>80 \%$ decrease) with progression of fibrosis being almost completely undetectable in livers with bridging fibrosis or established cirrhosis (Figure 2c).

\section{Pias3 expression increases with fibrosis progression and} significantly correlates with reduced Stat 3 DNA-binding As the most evident modification during fibrosis progression consisted in reduction of Stat3 DNA-binding activity, we examined whether changes in Socs3 and/or Pias3 expression might account for the additional inhibition of Stat3 DNAbinding. No significant variations in Socs3 mRNA expression were observed with progression of fibrosis (not shown). By contrast, an additional and steady increase in Pias3 mRNA levels occurred with progressing fibrosis. Compared to S1 livers Pias 3 mRNA increased by 1.8 -fold in $\mathrm{S} 2$ livers and by 2.4-fold in S3/4 livers (Figure 3a). Similarly, a steady increase in Pias3 protein expression was observed between $\mathrm{S} 1$ and S3/4 livers (Figure 3b). To further examine whether Pias3 could inhibit Stat3 DNA-binding, immunoprecipita-
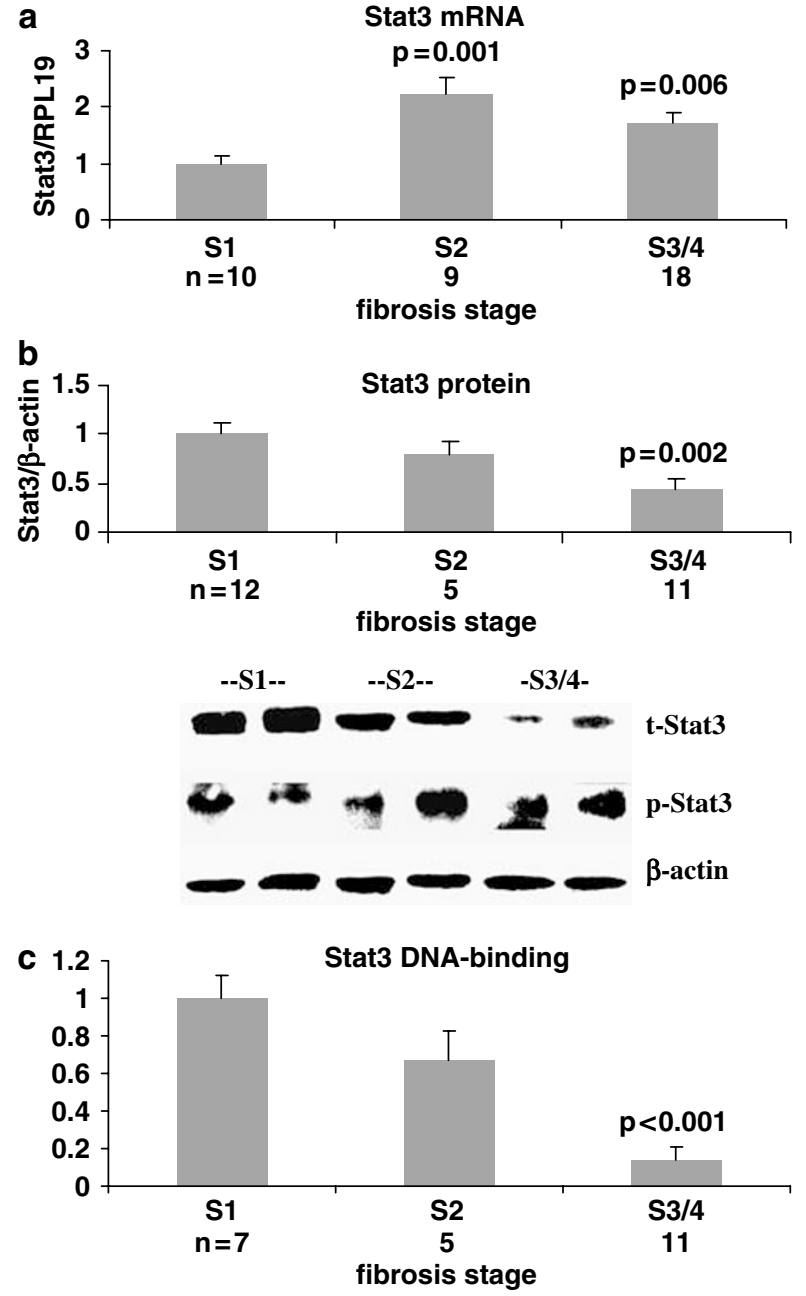

Figure 2 Expression of Stat3 mRNA, protein and DNA-binding activity in HCV-infected livers without fibrosis (S1), fibrotic septas extending from the portal tracts (S2) and bridging fibrosis and/or established cirrhosis (S3/4). Expression of all factors in HCV livers without fibrosis (S1) was set as 1 (100\%). Levels in HCV livers with fibrosis (S2, S3/4) are expressed as fold variation of values in HCV-livers without fibrosis (S1). (a) Expression of Stat3 mRNA by quantitative PCR normalized to their respective reporter gene values (riboprotein L19; RPL19). (b) Representative Western blots of total ( $t-S t a t 3)$ and phosphorylated ( $p$-Stat3) Stat3 as well as the densitometry analysis of total Stat 3 expression normalized to their respective loading controls ( $\beta$-actin). (c) Stat DNA-binding activity determined using a commercially available kit. The number of samples analyzed in each group $(n)$ is indicated below each column. The $P$-values at the top of the columns compare HCV livers with fibrosis ( $\mathrm{S} 2$ or S3/4) to HCV livers without fibrosis (S1). Represented are means \pm s.e.m.

tion experiments were performed. Interestingly, Stat3 coprecipitated

only weakly with Pias3 in HCV livers without fibrosis. By contrast, Pias3-Stat3 co-precipitation was found in immunoprecipitates from HCV livers with bridging fibrosis or cirrhosis (Figure 3c). This observation suggests that the formation of Pias3-Stat 3 complexes during fibrosis progression might explain a progressive decline in Stat3 activity by 
preventing the transcription factor from binding to its DNA-binding site. In addition, Pias3 mRNA expression significantly correlated with Stat3 DNA-binding activity (Table 3), which further sustains a strong relationship between Stat3 and Pias3 in HCV-infected livers progressing towards cirrhosis.

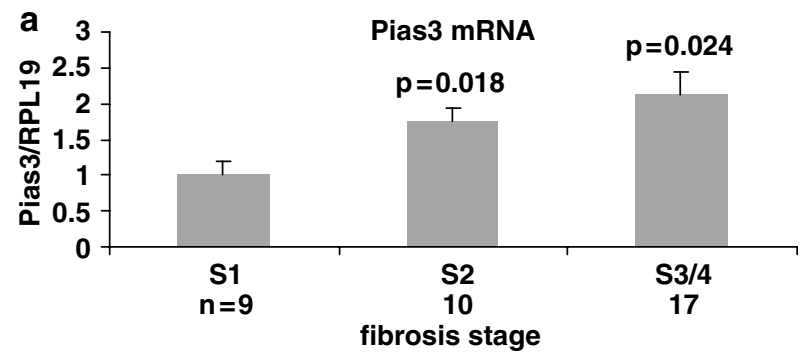

b

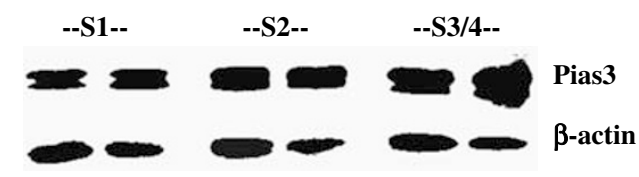

C

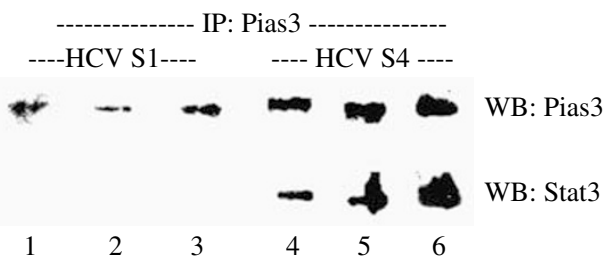

Figure 3 Expression of Pias 3 mRNA and protein in HCV-infected livers without fibrosis (S1), fibrotic septas extending from the portal tracts (S2) and bridging fibrosis and/or established cirrhosis (S3/4). Expression in HCV livers without fibrosis (S1) was set as $1(100 \%)$. Levels in HCV livers with fibrosis $(\mathrm{S} 2, \mathrm{~S} 3 / 4)$ are expressed as fold variation of values in HCV livers without fibrosis (S1). (a) Expression of Pias mRNA by quantitative PCR normalized to their respective reporter gene values (riboprotein L19; RPL19). The number of samples analyzed in each group $(n)$ is indicated below each column. The $P$-values at the top of the columns compare $\mathrm{HCV}$ livers with fibrosis (S2 or S3/4) to HCV livers without fibrosis (S1). Represented are means \pm s.e.m. (b) Representative Western blots Pias3 and their respective loading controls ( $\beta$-actin) showing high levels of Pias3 expression that slightly increase with fibrosis progression. (c) Immunoprecipitation-Western blot analysis of Pias3 in HCV livers without fibrosis (HCV S1) and with established cirrhosis (HCV S4). Membranes that were successively probed with Pias3 and Stat 3 antibodies show a strong co-precipitation of Pias3-Stat3 in livers with severe fibrosis.

\section{Decreased Hepatocyte Proliferation during Fibrosis Progression in HCV-Infected Livers Correlates with Reduced Stat3 Expression and Function}

Stat3 is though to play an important role in eliciting hepatocyte proliferation and liver repair after injury. ${ }^{12-15}$ In addition, there is currently no clear consensus whether liver fibrosis results from an alteration in the capacity of hepatocytes to proliferate or whether apoptosis constitutes the predominant phenomenon. We therefore studied the expression of two proliferation markers, cyclin D1 and PCNA at various stages of fibrosis and correlated them with Stat3 expression. Cyclin D1 is one of the earliest markers of cell cycle entry, whereas PCNA is activated during mid- to lateG1-phase of the cell cycle. Viral infection per se did not affect cyclin D1 and PCNA expression since no significant differences were found when HCV-negative normal livers were compared to HCV-infected livers without fibrosis (not shown). However, cyclin D1 and PCNA decreased significantly (by 25 and $50 \%$, respectively) with the onset of fibrosis. An additional reduction of cyclin D1 by more than $50 \%$ occurred in livers with bridging fibrosis or cirrhosis, whereas PCNA remained on constantly low levels throughout fibrosis progression (Figure 4a,b). Immunohistochemical studies revealed that PCNA was principally expressed in hepatocytes in nonfibrotic livers (Figure 5a) and occasionally in sinusoidal endothelial and Kupffer cells. The majority of the hepatocytes nuclei stained weakly to moderately positive for PCNA, whereas strong nuclear staining was only observed in a few hepatocytes. During fibrosis progression, PCNA was expressed essentially in hepatocytes and staining intensity decreased such as hardly any nuclear staining was found in livers with bridging fibrosis or cirrhosis (S3/4) (Figure 5b). Taken together, preferential PCNA expression in hepatocytes and the reduction of total PCNA and cyclin D1 levels quantified by Western blotting suggest a progressive alteration of hepatocyte proliferation during the fibrotic process. Owing to limited amount of tissue available from liver biopsies, paired analyses could only be performed for Stat3 protein expression and the protein expression of proliferation markers. As Stat3 DNA-binding and Stat3 protein expression decreased in a similar way during fibrosis progression, we used Stat 3 protein expression to examine potential correlation with protein expression of various markers assessed in the study. Interestingly, both proliferation markers signi-

Table 3 Correlations between different factors during fibrosis progression

\begin{tabular}{lccc}
\hline Factors & Correlation factor $(r)$ & $95 \%$ confidence interval & Level of significance ( $p$ ) \\
\hline Pias3 mRNA/Stat3 activity & $r=-0.5307$ & -0.8055 to -0.07169 & $P=0.023$ \\
Stat3 protein/cyclin D1 & $r=0.8012$ & 0.5233 to 0.9251 & $P<0.0001$ \\
Stat3 protein/PCNA & $r=0.8054$ & 0.5317 to 0.9268 & $P<0.0001$ \\
Stat3 protein/BCl-2 & $r=-0.4894$ & -0.7843 to -0.01414 & $P=0.0393$ \\
\hline
\end{tabular}



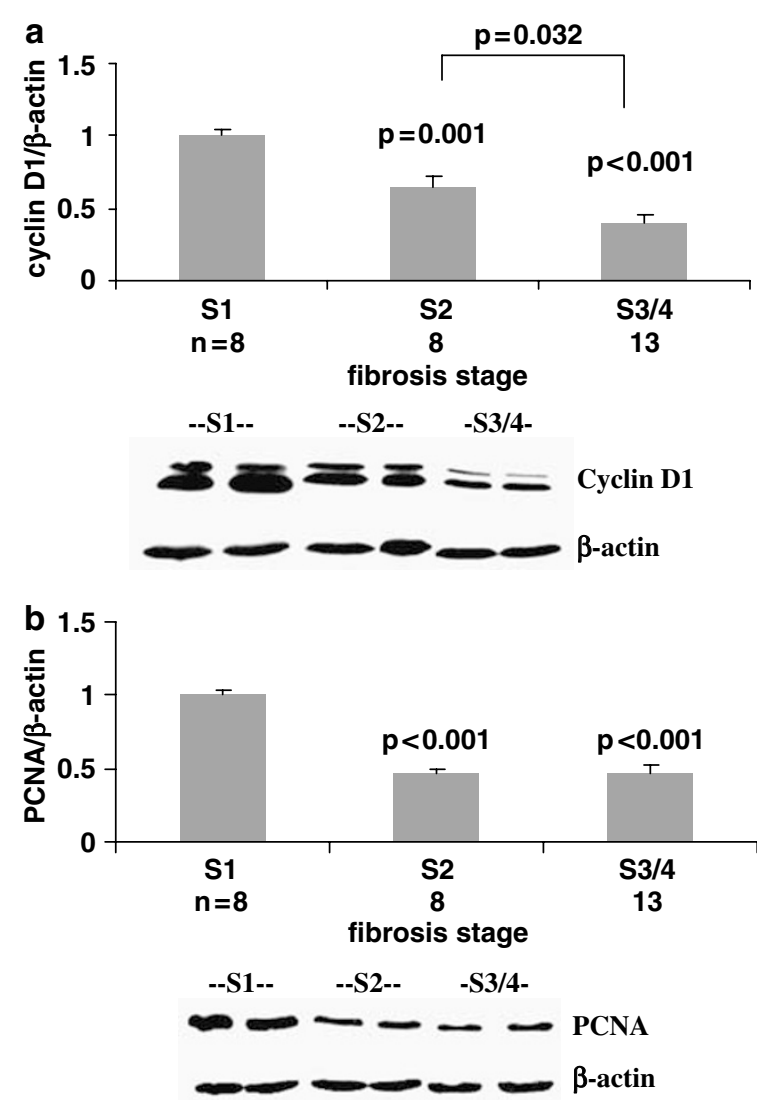

Figure 4 Expression of proliferation markers cyclin D1 and PCNA in HCVinfected livers without fibrosis (S1), fibrotic septas extending from the portal tracts (S2) and bridging fibrosis and/or established cirrhosis (S3/4). Expression of all factors in HCV livers without fibrosis (S1) was set as 1 (100\%). Levels in HCV livers with fibrosis (S2, S3/4) are expressed as fold variation of values in HCV livers without fibrosis (S1). (a) Representative Western blots and densitometry analysis of cyclin D1 normalized to their respective loading controls ( $\beta$-actin). (b) Representative Western blots and densitometry analysis of PCNA normalized to their respective loading controls ( $\beta$-actin). The number of samples analyzed in each group $(n)$ is indicated below each column. The $P$-values at the top of the columns compare HCV livers with fibrosis (S2 or S3/4) to HCV livers without fibrosis (S1). Represented are means \pm s.e.m.

ficantly and positively correlated with Stat 3 protein expression (Table 3) suggesting a link between downregulation of Stat 3 and reduced hepatocytes proliferation during fibrosis progression.

\section{Fibrosis Progression in HCV-Infected Livers is Associated with a Strong Antiapoptotic Profile in Infiltrating Inflammatory Cells}

In order to assess the potential role of apoptosis in the process of fibrosis progression, we next studied the expression of two principal markers, Bax and Bcl-2, regulating the pro- and antiapoptotic balance. Viral infection did not affect the expression of Bax and Bcl-2 since their levels of expression were similar in HCV-negative normal livers and HCVinfected livers without fibrosis (not shown). In addition, Bax expression did not vary with the progression of fibrosis

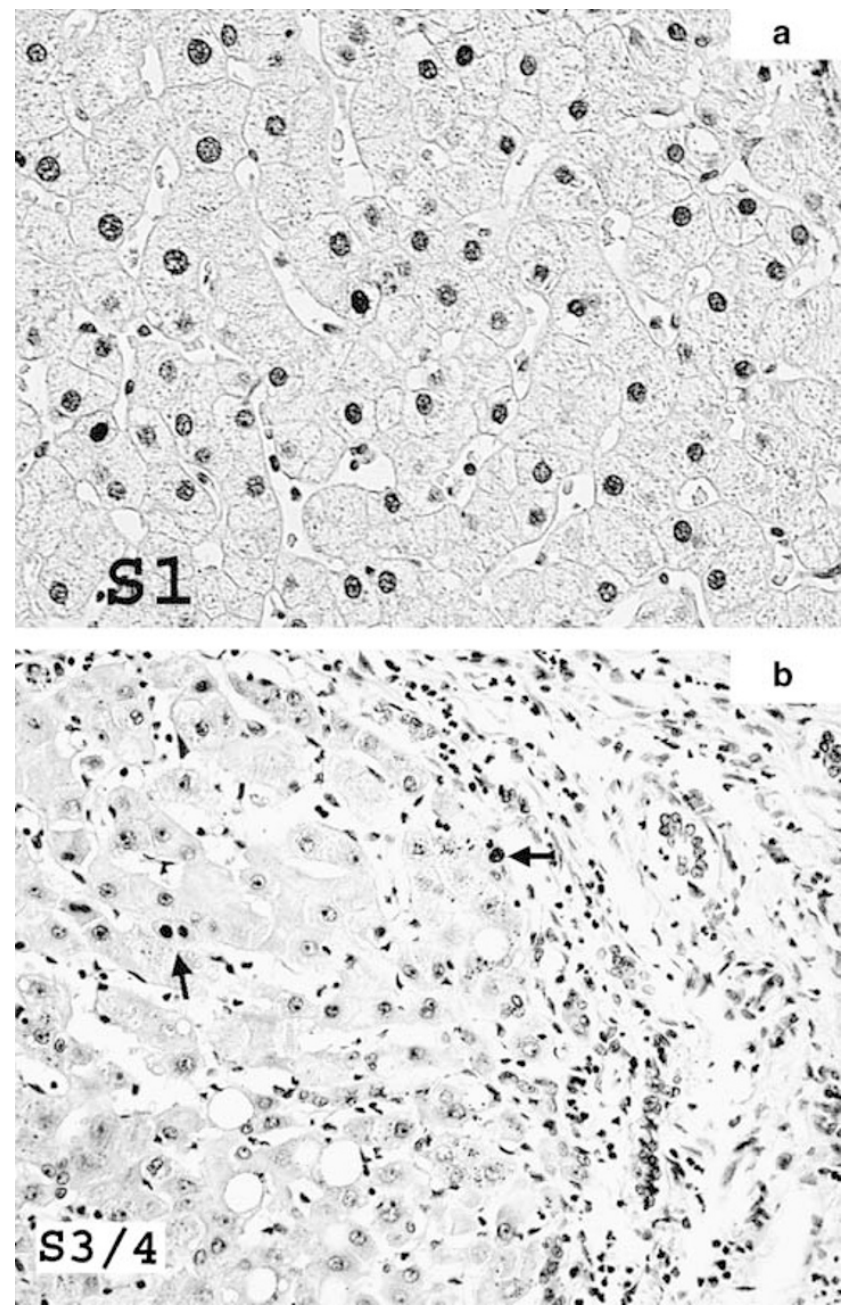

Figure 5 Representative immunohistochemical staining in HCV livers. (a) PCNA was moderately expressed mainly in the nuclei of hepatocytes with several nuclei staining strongly positive for PCNA in livers without fibrosis (S1) (magnification $\times 400$ ). (b) Hardly any PCNA-positive nuclei (arrows) were found in hepatocytes of livers with advanced fibrosis or cirrhosis $($ S3/4) (magnification $\times 200$ ).

(Figure 6a). Immmunohistochemical studies showed that Bax was principally expressed by biliary epithelial cells. No Bax staining was found in any other liver cell type, including hepatocytes and inflammatory cells (Figure 6b). Interestingly, $\mathrm{Bcl}-2$ expression started to increase with the onset of fibrosis and was strongly upregulated in S3/4 livers (Figure 7). Bcl-2 was expressed by portal inflammatory cells and by sinusoidal lymphocytes (Figure 8a,b) as well as endothelial and Kupffer cells. No Bcl-2 expression was found in hepatocytes, hepatic stellate cells or biliary epithelial cells. These results suggest a strong antiapoptotic response during fibrosis progression in inflammatory cells infiltrating the liver.

\section{DISCUSSION}

Several in vitro studies that have used the HCV replicon system suggest that HCV structural and nonstructural pro- 
a

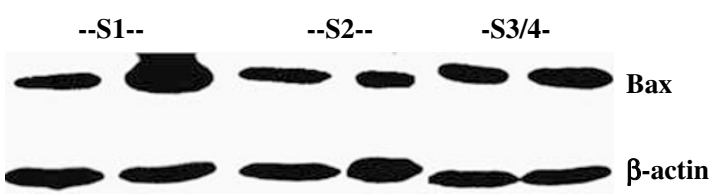

b

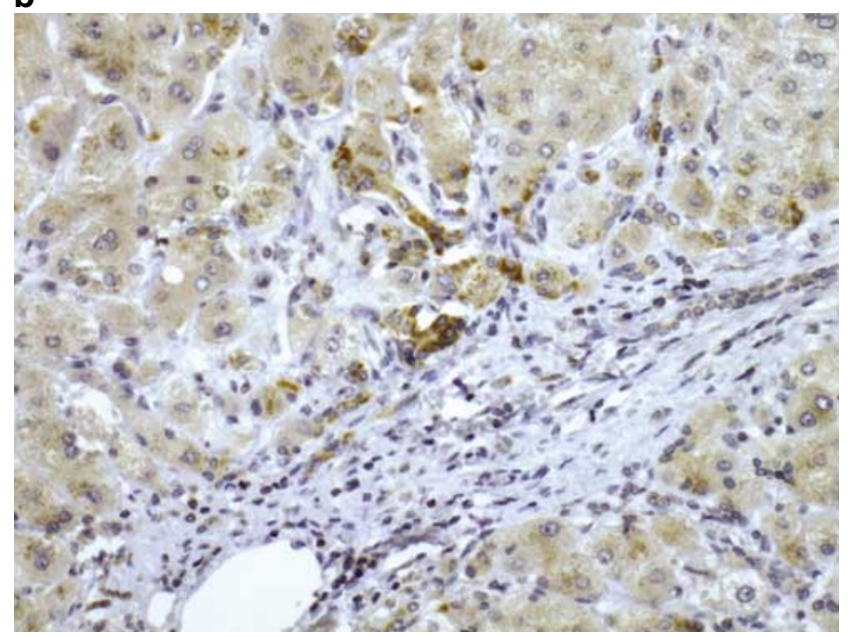

Figure 6 (a) Representative Western blots of proapoptotic bax compared to their respective loading controls ( $\beta$-actin). Bax protein expression did no change significantly in samples without fibosis (S1) compared to samples with moderate (S2) or advanced fibrosis and cirrhosis (S3/4). (b) Representative immunohistochemical staining (magnification $\times 400$ ) of Bax in HCV livers. Bax was principally expressed in proliferating bile ducts (dark brown staining) in livers with severe fibrosis. No immunostaining was observed in hepatocytes or inflammatory cells of the liver.

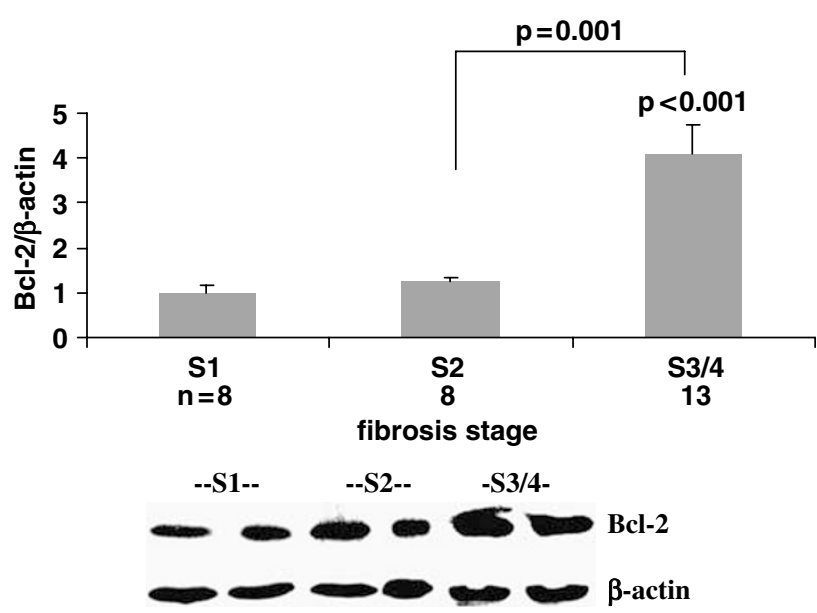

Figure 7 Representative Western blots and densitometry analysis of antiapoptotic Bcl-2 normalized to their respective loading controls ( $\beta$-actin) in $\mathrm{HCV}$-infected livers without fibrosis (S1), fibrotic septas extending from the portal tracts (S2) and bridging fibrosis and/or established cirrhosis (S3/4). The number of samples analyzed in each group $(n)$ is indicated below each column. The $P$-values at the top of the columns compare HCV livers with fibrosis (S2 or S3/4) to HCV livers without fibrosis (S1). Represented are means \pm s.e.m.

teins interfere with Stat3 activation and signaling. ${ }^{1,3,25-27}$ Our group has also shown that Stat3 activity is altered in vivo in human alcoholic and HCV end-stage liver disease. ${ }^{18,19}$
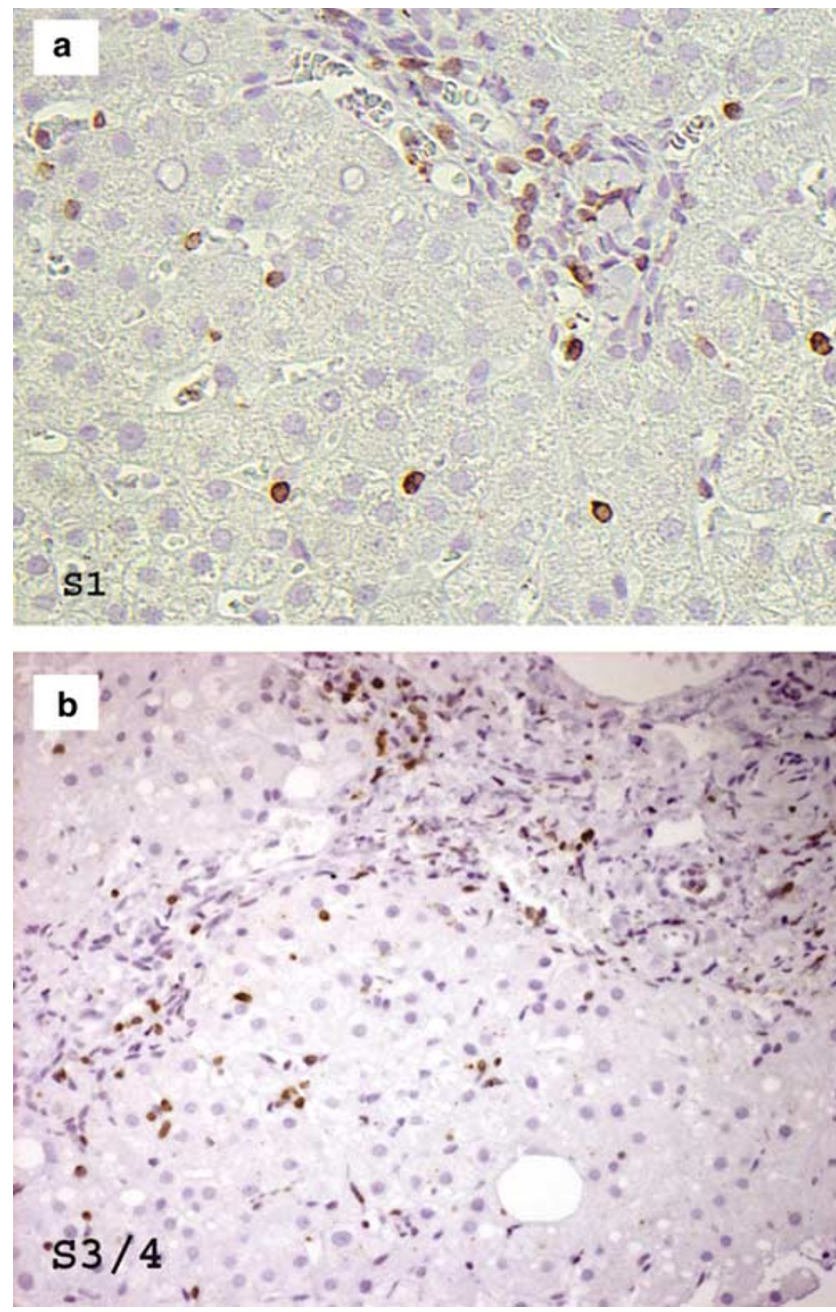

Figure 8 Representative immunohistochemical staining in HCV livers. (a) $\mathrm{BCl}-2$ staining was principally restricted to inflammatory cells of the portal tracts (brown staining) and some rare lymphocytes in the sinusoids in livers without fibrosis (S1) (magnification $\times 400$ ). (b) Staining intensity and the number of $\mathrm{BCl}-2$-positive cells increased but remained restricted to inflammatory cells (dark brown staining) in livers with advanced fibrosis or cirrhosis $($ S3/4) (magnification $\times 200)$.

Although one can conclude from these observations that Stat 3 activity is altered by infection with HCV, it remains to be established whether inhibition of Stat 3 activity plays a role in disease progression in vivo in humans and how this alteration relates to the proliferation-apoptosis status in HCV liver samples with various levels of fibrosis. The present study shows that HCV in human liver samples in vivo, induces an alteration in Stat3 function that worsens with fibrosis progression leading to an almost complete Stat 3 inhibition at the stage of cirrhosis. The progressive inhibition of Stat 3 correlates significantly with a gradual increase in Pias3 expression and formation of Stat3-Pias3 protein complexes preventing the transcription factor from binding its DNAbinding site. In addition, progressive inhibition of Stat3 DNA-binding during fibrosis progression significantly cor- 
relates with a reduced proliferative capacity of hepatocytes. Our findings are sustained by recent data that showed altered Stat3 activation in HCV-infected livers presumably related to reduced Stat3 expression. ${ }^{28}$ These observations are in contradiction to HCV replicon experiments where expression of $\mathrm{HCV}$ proteins generally induced Stat 3 expression and activity. ${ }^{3,26,27}$ However, replicons are usually established in immortalized tumor cells that might behave different than differentiated hepatocytes in vivo in humans. ${ }^{29}$

Pias3, a known inhibitor of Stat 3 DNA-binding, ${ }^{22}$ is upregulated in HCV livers compared to normal noninfected livers and further increases during fibrosis progression. Immunoprecipitation studies show no or weak Stat3-Pias3 interaction in normal and HCV livers without fibrosis which might explain why Stat3 DNA-binding activity is preserved in normal livers and only slightly affected in nonfibrotic HCVinfected livers. In addition, a strong Stat3-Pias3 co-precipitation is found in livers with bridging fibrosis or cirrhosis suggesting that inhibition of Stat3 DNA-binding activity is closely related to an increase in Stat3-Pias3 interaction. This point is further sustained by a significant inverse correlation between Pias3 expression and Stat3 DNA-binding. A similar observation, that is, preserved Stat phosphorylation and decreased Stat DNA-binding activity, was made for Stat1-Pias1 in a transgenic mice expressing HCV proteins and in liver biopsies of HCV-infected patients. ${ }^{30,31}$

Several confounding factors that might have affected Stat 3 activity need to be acknowledged. As Stat 3 might be influenced by the inflammatory state, only liver biopsies with low inflammation scores were chosen to reduce the role of inflammatory changes as a potential confounder in our analysis. The interferon-mediated intracellular antiviral response to HCV might also influence the activity of Stat proteins. Especially, interferon-induced activation of Stat 1 might increase SOCS3 levels that in turn downregulate Stat3 phosphorylation and activity. ${ }^{32-34}$ It is, however, unlikely that this mechanism accounts for the progressive alteration in Stat3 DNA-binding activity in our study since no major changes in SOCS3 and phospho-Stat3 levels are found in HCV-infected livers at various stages of fibrosis.

To gain some insight in the functional consequences of alterations in Stat3 function on the proliferation-apoptosis balance, we next examined the expression of proliferation and apoptosis markers at different stages of liver fibrosis and correlated them with Stat3 expression. Fibrosis progression is associated with reduced cellular proliferation as indicated by a highly significant downregulation of cyclinD1 and PCNA expression. In addition, a highly significant correlation is found between both markers and Stat 3 expression. Stat 3 is known to play a role in hepatocytes proliferation in rodents. ${ }^{17,35}$ Inappropriate constitutive activation of Stat 3 does also occur in numerous human cancers. ${ }^{36}$ In a previous report, we have shown that Stat 3 is mainly expressed in hepatocytes and small bile ducts in human livers. ${ }^{18} \mathrm{Im}$ munohistochemical data in this study reveals that PCNA is principally expressed in hepatocytes indicating that the reduction in expression of proliferation markers mainly reflects reduced hepatocytes proliferation. Given the preferential expression of Stat 3 and PCNA in hepatocytes, it is tempting to link decreased hepatocytes proliferation to decreased Stat3 activity in HCV livers with progressive fibrosis. This hypothesis is in line with data obtained in HCV replicon experiments where the HCV core protein directly activated Stat 3 which in turn resulted in cellular proliferation through upregulation of cyclin D. ${ }^{3}$ In addition, Stat 3 is thought to be involved in initiating intracellular antiviral pathways and defense mechanisms. ${ }^{5,6,37}$ Reduced Stat3 function might therefore favor viral persistence that potentiates liver damage which, together with impaired repair mechanisms, might lead to fibrosis progression.

Surprisingly, progressive fibrosis is also associated with a positive overall antiapoptotic response. This positive antiapoptotic balance results primarily from increasing Bcl-2 levels. Stimulation of the antiapoptotic Bcl-2 signaling pathway as well as Bcl-2 overexpression have been reported at late stages of fibrosis progression in HCV-infected livers. ${ }^{38-40}$ However, it remains uncertain whether and how Bcl-2 may be involved in promoting liver damage. In the present study, immunohistochemistry revealed that $\mathrm{Bcl}-2$ is principally expressed by inflammatory cells of the portal tracts and by lymphocytes in the sinusoids. The absence of Bcl-2 in hepatocytes excludes a protective effect on hepatocytes. In addition, proapoptotic Bax does not seem to play a major regulatory role in hepatocytes or inflammatory cells for its overall expression does not change significantly and is principally restricted to proliferating cholangioles in an advanced stage of the disease. As hepatitis $\mathrm{C}$ is mainly an immunedriven disease without a direct cytotoxic effect of the virus, an antiapoptotic response in infiltrating inflammatory cells may favor the persistence of potentially cytotoxic lymphocytes aiming to clear infected hepatocytes and thereby increase liver damage. Together with a progressive decrease in Stat3-mediated proliferation and repair mechanisms of the remaining hepatocytes, fibrosis may occur and progress once a critical threshold keeping the system in balance has been passed.

Based on our studies and data reported in HCV replicon studies, we propose the following mechanism that may contribute, at least partially, to fibrosis progression in HCVinfected patients:

1. HCV infection induces a Pias3-mediated alteration of Stat 3 activity.

2. Disturbed activation of Stat3-dependent intracellular signaling cascades leads to a reduced proliferation capacity of hepatocytes and may also alter the initiation of intracellular antiviral defense pathways.

3. A progressive Bcl-2-dependent switch towards an antiapoptotic profile in infiltrating inflammatory cells enhances survival of potentially cytotoxic cells that in turn aggravate liver damage. 


\section{ACKNOWLEDGEMENT}

We are indebted to Dr Christine Sempoux for her help in performing the immunohistochemistry analysis and her expertise in scoring the liver samples.

1. Hosui A, Ohkawa $K$, Ishida $H$, et al. Hepatitis $C$ virus core protein differently regulates the Jak-Stat signaling pathway under interleukin6 and interferon-gamma stimuli. J Biol Chem 2003;278:28562-28571.

2. Waris G, Tardif KD, Siddiqui A. Endoplasmic reticulum (ER) stress: hepatitis $C$ virus induces an ER-nucleus signal transduction pathway and activates NF-kappaB and Stat-3. Biochem Pharmacol 2002;64:1425-1430.

3. Yoshida $T$, Hanada $T$, Tokuhisa $T$, et al. Activation of Stat3 by the hepatitis $C$ virus core protein leads to cellular transformation. J Exp Med 2002;196:641-653.

4. Gong G, Waris G, Tanveer R, et al. Human hepatitis C virus NS5A protein alters intracellular calcium levels, induces oxidative stress, and activates Stat-3 and NF-kappa B. Proc Natl Acad Sci USA 2001;98: 9599-9604.

5. Basu A, Meyer K, Lai KK, et al. Microarray analyses and molecular profiling of Stat3 signaling pathways induced by hepatitis $\mathrm{C}$ virus core protein in human hepatocytes. Virology 2006;349:347-358.

6. Ehrmann Jr J, Aiglova K, Ehrmann J, et al. Expression of JAK, STAT, SOCS and PIAS in liver biopsies of patients with chronic hepatitis C: relationship to grading/staging and response to combined interferon therapy. J Hepatol 2006;44:S212 (abstact).

7. Ghosh AK, Steele R, Meyer K, et al. Hepatitis C virus NS5A protein modulates cell cycle regulatory genes and promotes cell growth. J Gen Virol 1999:80:1179-1183.

8. Shackel NA, McGuinness $\mathrm{PH}$, Abbott $\mathrm{CA}$, et al. Insights into pathobiology of hepatitis $C$ virus-associated cirrhosis: analysis of intrahepatic differential gene expression. Am J Pathol 2002;160: 641-654.

9. Papakyriakou P, Tzardi M, Valatas V, et al. Apoptosis and apoptosis related proteins in chronic viral liver disease. Apoptosis 2002;7: 133-141.

10. Floreani A, Guido M, Bortolami M, et al. Relationship between apoptosis, tumor necrosis factor, and cell proliferation in chronic cholestasis. Dig Liver Dis 2001;33:570-575.

11. Farinati $F$, Cardin R, Fiorentino $M$, et al. Imbalance between cytoproliferation and apoptosis in hepatitis $\mathrm{C}$ virus related chronic liver disease. J Viral Hepat 2001;8:34-40.

12. Strain AJ, Diehl AM (eds). Liver Growth and Repair. Chapman \& Hall: London, 1998.

13. Taub R. Hepatoprotection via the IL-6/Stat3 pathway. J Clin Invest 2003;112:978-980.

14. Costa RH, Kalinichenko VV, Holterman AX, et al. Transcription factors in liver development, differentiation, and regeneration. Hepatology 2003;38:1331-1347.

15. Mangnall D, Bird NC, Majeed AW. The molecular physiology of liver regeneration following partial hepatectomy. Liver Int 2003;23:124-138.

16. Yamada Y, Kirillova I, Peschon JJ, et al. Initiation of liver growth by tumor necrosis factor: deficient liver regeneration in mice lacking type I tumor necrosis factor receptor. Proc Natl Acad Sci USA 1997;94: 1441-1446.

17. Cressman DE, Greenbaum LE, DeAgelis RA, et al. Liver failure and defective hepatocyte regeneration in interleukin-6-deficient mice. Science 1996;274:1379-1383.

18. Starkel P, Bishop K, Horsmans $Y$, et al. Expression and DNA-binding activity of signal transducer and activator of transcription 3 in alcoholic cirrhosis compared to normal liver and primary biliary cirrhosis in humans. Am J Pathol 2003;162:587-596.
19. Stärkel P, De Saeger C, Leclercq I, et al. Deficient Stat3 DNA-binding is associated with high Pias3 expression and a positive anti-apoptotic balance in human end-stage alcoholic and hepatitis $C$ cirrhosis. J Hepatol 2005;43:687-695.

20. Batts KP, Ludwig J. Chronic hepatitis. An update on terminology and reporting. Am J Surg Pathol 1995;19:1409-1417.

21. Picard C, Lambotte L, Starkel P, et al. Retrorsine: a kinetic study of its influence on rat liver regeneration in the portal branch ligation model. J Hepatol 2003;39:99-105.

22. Chung CD, Liao J, Liu B, et al. Specific inhibition of Stat3 signal transduction by PIAS3. Science 1997;278:1803-1805.

23. Gisselbrecht $\mathrm{S}$. The CIS/SOCS proteins: a family of cytokine-inducible regulators of signaling. Eur Cytokine Network 1999;10:463-470.

24. Yasukawa $\mathrm{H}$, Sasaki A, Yoshimura A. Negative regulation of cytokine signaling pathways. Annu Rev Immunol 2000;18:143-164.

25. Heim MH, Moradpour D, Blum HE. Expression of hepatitis $C$ virus proteins inhibits signal transduction through the Jak-Stat pathway. J Virol 1999;73:8469-8475.

26. Sarcar B, Ghosh AK, Steele R, et al. Hepatitis C virus NS5A mediated Stat3 activation requires co-operation of Jak1 kinase. Virology 2004;322:51-60.

27. Waris G, Turkson J, Hassanein T, et al. Hepatitis C virus (HCV) constitutively activates Stat-3 via oxidative stress: role of Stat-3 in HCV replication. J Virol 2005;79:1569-1580.

28. Larrea $\mathrm{E}$, Aldabe $\mathrm{R}$, Molano $\mathrm{E}$, et al. Altered expression and activation of STATs (signal transduction and activator of transcription in HCV infection: in vivo and in vitro studies. Gut 2006;55:1188-1196.

29. Bartenschlager $R$. The hepatitis $C$ virus replicon system: from basic research to clinical application. J Hepatol 2005;43:210-216.

30. Blindenbacher A, Duong FH, Hunziker $L$, et al. Expression of hepatitis $c$ virus proteins inhibits interferon alpha signaling in the liver of transgenic mice. Gastroenterology 2003;124:1465-1475.

31. Duong $F H$, Filipowicz $M$, Tripodi $M$, et al. Hepatitis $C$ virus inhibits interferon signaling through up-regulation of protein phosphatase $2 \mathrm{~A}$. Gastroenterology 2004;126:263-277.

32. Dumoulin FL, Wennrich U, Nischalke HD, et al. Intrahepatic mRNA levels of interferon gamma and tumor necrosis factor alpha and response to antiviral treatment of chronic hepatitis C. J Hum Virol 2001;4:195-199.

33. Bode JG, Ludwig S, Ehrhardt C, et al. IFN-alpha antagonist activity of $\mathrm{HCV}$ core protein involves induction of suppressor of cytokine signaling-3. FASEB J 2003;17:488-490.

34. Walsh MJ, Jonsson JR, Richardson MM, et al. Non-response to antiviral therapy is associated with obesity and increased hepatic expression of suppressor of cytokine signaling 3 (SOCS-3) in patients with chronic hepatitis C, viral genotype 1. Gut 2006;55:529-535.

35. Li W, Liang $X$, Kellendonk $C$, et al. STAT3 contributes to the mitogenic response of hepatocytes during liver regeneration. J Biol Chem 2002:277:28411-28417.

36. Bowman T, Garcia R, Turkson J, et al. Stats in oncogenesis. Oncogene 2000;19:2474-2488

37. Fimia GM, Evangelisti $C$, Alonzi $T$, et al. Conventional protein kinase $C$ inhibition prevents alpha interferon-mediated hepatitis $C$ virus replicon clearance by impairing Stat activation. J Virol 2004;78: 12809-12816.

38. Smith MW, Yue ZN, Korth MJ, et al. Hepatitis C virus and liver disease: global transcriptional profile and identification of potential markers. Hepatology 2003;38:1458-1467.

39. Tsamandas AC, Thomopoulos K, Zolota V, et al. Potential role of bcl-2 and bax mRNA and protein expression in chronic hepatitis type $B$ and C: a clinicopathologic study. Mod Pathol 2003;16:1273-1288.

40. Nakopoulou L, Stefanaki K, Vourlakou C, et al. Bcl-2 protein expression in acute and chronic hepatitis, cirrhosis and hepatocellular carcinoma. Pathol Res Pract 1999;195:19-24. 